\title{
Instrument independent diffuse reflectance spectroscopy
}

\author{
Bing Yu, Henry L. Fu, and Nirmala Ramanujam \\ Duke University, Department of Biomedical Engineering, Durham, North Carolina 27708
}

\begin{abstract}
Diffuse reflectance spectroscopy with a fiber optic probe is a powerful tool for quantitative tissue characterization and disease diagnosis. Significant systematic errors can arise in the measured reflectance spectra and thus in the derived tissue physiological and morphological parameters due to real-time instrument fluctuations. We demonstrate a novel fiber optic probe with real-time, self-calibration capability that can be used for UVvisible diffuse reflectance spectroscopy in biological tissue in clinical settings. The probe is tested in a number of synthetic liquid phantoms over a wide range of tissue optical properties for significant variations in source intensity fluctuations caused by instrument warm up and day-to-day drift. While the accuracy for extraction of absorber concentrations is comparable to that achieved with the traditional calibration (with a reflectance standard), the accuracy for extraction of reduced scattering coefficients is significantly improved with the self-calibration probe compared to traditional calibration. This technology could be used to achieve instrument-independent diffuse reflectance spectroscopy in vivo and obviate the need for instrument warm up and post/premeasurement calibration, thus saving up to an hour of precious clinical time. $\odot 2011$ Society of Photo-Optical Instrumentation Engineers (SPIE). [DOI: 10.1117/1.3524303]
\end{abstract}

Keywords: diffuse reflectance spectroscopy; fiber optics sensors; tissue phantoms.

Paper 10116SSRR received Mar. 8, 2010; revised manuscript received Aug. 31, 2010; accepted for publication Sep. 2, 2010; published online Jan. 20, 2011.

\section{Introduction}

Fiber-optic-based diffuse reflectance spectroscopy (DRS) is a nondestructive technique that is sensitive to tissue physiological and morphological composition. In combination with Monte Carlo $^{1-4}$ or diffusion-based models, ${ }^{5-7}$ this approach provides quantitative information, specifically the wavelength-dependent absorption and scattering properties from which the underlying tissue physiological and morphological information can be derived. Quantitative diffuse reflectance spectroscopy has recently been evaluated for precancer detection and cancer diagnostics, ${ }^{2,5,8-18}$ intraoperative tumor margin assessment, ${ }^{19-21}$ monitoring of tumor response to therapy, ${ }^{19,22-24}$ and tissue oximetry. ${ }^{25}$ Endpoints of the quantitative analysis that solely reflect "tissue" characteristics can be compared across different patient studies measured with different instruments. This quantitative DRS approach facilitates consensus on methods for validation and translation, and widens the acceptance of this technique for biomedical applications.

Calibration is a critical step in insuring quality control prior to quantitative model-based analysis of diffuse reflectance spectra. It accounts for the spectral power distribution of the light source, wavelength-dependent response of the instrument, and throughput of the fiber optic probe. Commonly used calibration methods correct for instrument-dependent throughput by dividing the raw tissue spectra by a calibration spectrum measured on a spectrally flat diffuse reflectance standard, such as the spectralon reflectance standard SRS-99 (referred to as Spectralon) from Labsphere, Incorporated (North Sutton, New Hampshire),

Address all correspondence to: Bing Yu, Department of Biomedical Engineering, Duke University, 136 Hudson Hall, Box 90281, Durham, NC 27708-0281. Tel: 919-660-5032; Fax: 919-684-4488. E-mail: bing.yu@duke.edu. and/or a tissue phantom. $3,5,8,9,13,18,26$ The calibration measurements are typically performed before or after the clinical measurements of all samples. With these calibration methods, however, there could potentially be significant errors in the measured reflectance spectra, and consequently the extracted tissue absorption and scattering properties. A few sources of these errors include real-time intensity fluctuations, fiber bending-induced loss, and variations in the coupling between the fiber optic probe and calibration reflectance standard. Current calibration approaches require at least 20-40 min for instrument warm-up to eliminate significant lamp intensity fluctuations, and another 5 to 20 min (depending on whether tissue phantoms are used) for calibration measurements in the clinic.

We recently demonstrated the feasibility of performing quantitative DRS in the UV-visible band using a self-calibration (SC) fiber optic probe. ${ }^{27}$ The probe has two completely separate channels, one for tissue spectroscopy and the other for calibration of the tissue spectral signal. The reference spectrum is collected by the built-in calibration channel at the same time as the tissue measurement, and can be used to replace calibration measurements that need to be performed immediately before or after collecting the tissue spectra. It was shown that the lamp intensity fluctuations [which were simulated by inserting a neutral density (ND) filter between the lamp and illumination fibers after the lamp had been warmed-up] could be reduced from $6 \mathrm{~dB}$ down to $\pm 0.13 \mathrm{~dB}$ (or $\pm 3 \%$ ) using this built-in self-calibration channel. The probe was tested in a number of liquid phantoms over a range of tissue optical properties after instrument warm up. Absorption and scattering coefficients were extracted with an average absolute error and standard deviation of $6.9 \pm 7.2 \%$ and $3.5 \pm 1.5 \%$, respectively, with an inverse scalable Monte Carlo

1083-3668/2011/16(1)/011010/12/\$25.00 @ 2011 SPIE 
(MC) model developed by our group. ${ }^{1}$ The errors for extraction of phantom absorption and scattering coefficients were comparable with those achieved in a previous study by our group with the traditional Spectralon-based calibration using two different instruments and phantoms with similar optical properties. ${ }^{28}$ The results from this original publication demonstrated the concept of using a SC probe in lieu of a traditional diffuse reflectance standard for instrument calibration in laboratory or clinic settings.

Although the previously developed SC probe (referred to as SC probe A) achieved excellent accuracy in measuring phantom optical properties after the lamp warm-up period, it was not effective in accounting for spatial variations in the lamp intensity that occur during lamp warm-up. This is because the spatial distribution of the lamp emission varies significantly during warm-up, which results in time-varying ratios in the light power coupled into the independent sensing and calibration channels. Thus with SC probe A, it was not possible to test the effect of self-calibration during lamp warm-up due to the time-varying intensity ratio between the sensing and calibration channels. Instead, ND filters were used. In this work, we report an improved self-calibration probe (referred to as $\mathrm{SC}$ probe B) design that builds on the previously described proof-of-concept probe to provide effective real-time calibration during the lamp warm-up period, which can be as long as $30 \mathrm{~min}$. Instead of using two independent source fibers for the two channels, this new design uses an optical fiber splitter (coupler) as a source fiber that overcomes the time-varying differences in the power coupled into the two channels. Using SC probe B, different sources of systematic error that can give rise to variations in source intensity, which serves as the basis for the prior probe design, were characterized. In this work, we show that the effect of lamp intensity fluctuations during real warm-up can be reduced from $1.0 \mathrm{~dB}$ (or $20 \%$ ) to less than $0.04 \mathrm{~dB}$ (or $1 \%$ ) with the use of the new probe design, which was not possible with the previous probe design (SC probe A) or traditional calibration methods. In this work, phantom studies covering a wide range of optical properties were performed under conditions that are representative of a practical clinical setting in which there were significant source intensity fluctuations to demonstrate the practical performance of the DRS system. The results obtained indicate that using the self-calibration approach provides excellent accuracy for quantitative DRS under conditions of significant source intensity fluctuations that cannot be corrected with the previous probe design (SC probe A) or traditional calibration methods. The improved design of self-calibration probe design B allows for this effective calibration.

\section{Method and Materials}

\subsection{Instrumentation}

The UV-VIS DRS system, as depicted in Fig. 1(a), consists of a 450-W xenon lamp as the broadband light source (FL1039, HORIBA Jobin Yvon, New Jersey), a laboratory-made self-calibration fiber optic probe to deliver illumination light to and collect diffusely reflected light from the tissue sample, and an imaging spectrometer with a 2-D charge-coupled decide (CCD) camera (iHR-320, HORIBA Jobin Yvon, New Jersey). The fiber optic probe has two channels: a tissue sensing channel (referred to as tissue $\mathrm{CH}$ ) to collect a diffuse reflectance spectrum from a tissue sample, and a calibration channel (referred to as $\mathrm{Cal} \mathrm{CH}$ ) to collect a calibration spectrum concurrently with the tissue measurement, which can be used for real-time instrument and probe calibration. The probe uses a large core $(400 \mu \mathrm{m}) 1 \times 2$ fiber splitter (Fiber Optic Network Technology Company, British Columbia, Canada) as the source fiber for both sample illumination and self-calibration (the red fibers in Fig. 1). The light launched into the common arm of the splitter is divided into two channels, $80 \%$ to the tissue channel and $20 \%$ to the calibration channel. At the probe tip, eight $200-\mu \mathrm{m}$ (core diameter) detection fibers (the blue fibers in Fig. 1) form a ring around the $400-\mu \mathrm{m}$ tissue illumination fiber and collect the diffusely reflected light from the sample. The center-to-center distances between the single illumination fiber and the eight detection fibers range between 690 and $860 \mu \mathrm{m}$. The self-calibration illumination fiber terminates inside the rigid part of the probe tip, as shown in Fig. 1(b), and a portion of the calibration light is specularly reflected by the polished end surface of a stainless steel rod and coupled back into a $200-\mu \mathrm{m}$ (core diameter) calibration return fiber (the green fiber in Fig. 1). Since the proximal ends of the calibration fibers and stainless steel rod are aligned and permanently fixed together inside a short piece of stainless steel capillary tube using epoxy, as shown in Fig. 1(b), the relative angles, distance, and spacing of the two fibers and the rod are all fixed. The reflector does not have to be a specular reflector, and any reflector can be used as long as it provides a comparable signal level as the tissue channel. All 200- $\mu \mathrm{m}$ fibers are made of identical silica/silica, high -OH optical fibers with numerical apertures (NA) of 0.22 (Polymicro Technologies, Phoenix, Arizona) to ensure the same bending response. This new probe design guarantees that the two channels respond to changes of the lamp emission intensity in the same manner.

In the detection fiber bundle, the detection fibers and the calibration return fiber are arranged into two columns, with two rows of dead fibers of the same diameter (the white fibers) for spacing between the two channels, as shown in Fig. 1(a). The fiber bundle is coupled to the imaging spectrograph iHR-320 via a reflective optics coupler with a 1:1.2 magnification. The collected diffuse reflectance and calibration beams are diffracted and projected onto different areas of the CCD camera and recorded by the laptop computer. The CCD chip has 1024 (horizontal) $\times$ 256 (vertical) pixels with a pixel size of $26 \times 26 \mu \mathrm{m}$. The vertical axis is used to separate the self-calibration channel and the sensing channel, while the horizontal axis is used to detect the diffracted incident light. Because the spectrometer is optimized for spectroscopy (the CCD is in the tangential focal plane), relatively large spherical aberrations were measured in the vertical direction of the CCD. This means the image of an active fiber on the CCD in the vertical direction is larger than the diameter of the fiber. Therefore, two dead fibers were used to separate the two channels on the CCD, as indicated in Fig. 1(a). Pixels 62 to 92 along the vertical axis are binned for the self-calibration channel, while pixels 96 to 168 are binned for the sensing channel with three pixels left for spacing between the two channels, with no measurable cross talk on the CCD.

Each scan of the spectrometer covered only $128.7 \mathrm{~nm}$. The input slit width of the spectrometer was set to $0.6 \mathrm{~mm}$, resulting in a wavelength resolution of $1.4 \mathrm{~nm}$. A complete spectrum from 


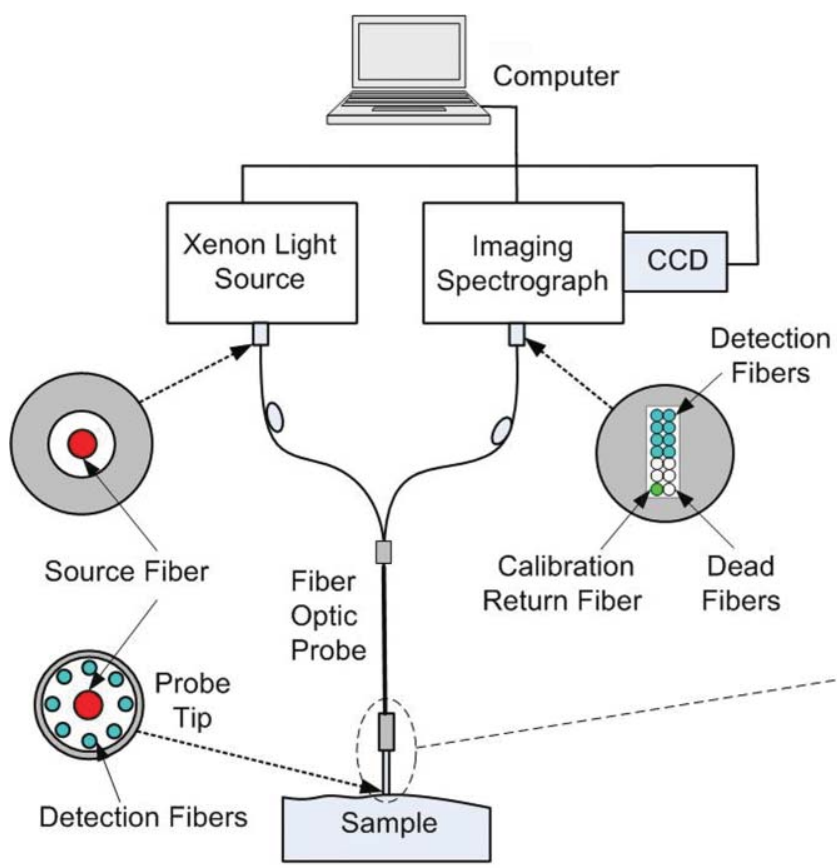

(a)

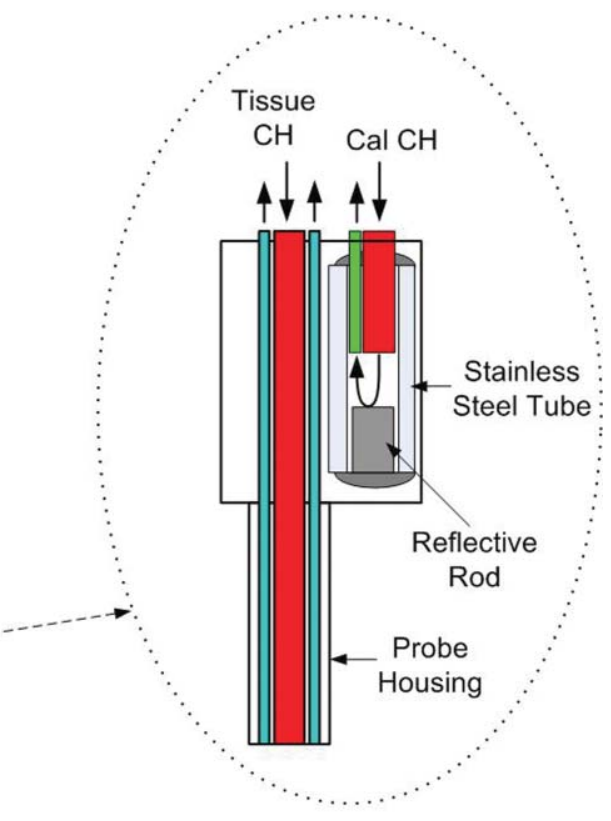

(b)

Fig. 1 Schematic of (a) the UV-VIS DRS system with a splitter-based self-calibration fiber optic probe, and (b) the probe tip showing the termination of the fibers inside the probe housing. The detection fibers and calibration return fibers in the detection bundle are imaged onto the 2-D CCD as two separate tracks. (Color online only.)

350 to $600 \mathrm{~nm}$ took two scans with a low scan covering 349.4 to $479.8 \mathrm{~nm}$ and a high scan covering 472.2 to $600.9 \mathrm{~nm}$. In addition to maintaining high wavelength resolution, acquiring two separate scans for the low and high wavelength ranges enabled the use of different integration times to achieve comparable signal for the entire wavelength range.

\subsection{Characterizing Different Sources of Systematic Errors}

Three major sources of errors, including day-to-day variations in intensity, lamp warm-up, and fiber bending loss, were measured with the DRS system and SC probe B. First, day-to-day variations in lamp intensity were characterized under the same experimental conditions by taking five consecutive scans from the same Spectralon reflectance standard (Spectralon) after a lamp warm-up period of $60 \mathrm{~min}$ (to remove the effect of instrument warm-up drift as a variable) per day on each of two different days. The same experimental condition means that the instrument, probe-instrument interface, probe-Spectralon coupling, and fiber bending were unchanged between the two different days. Second, the effect of lamp warm-up was characterized by acquiring spectra from the same Spectralon using SC probe $\mathrm{B}$ at 13 time points between 2 and 60 min after the lamp was turn on for each of two off/on cycles. The intensity counts at two wavelengths of 475 and $575 \mathrm{~nm}$ were plotted against time to show lamp intensity fluctuations during warm-up (the intensity fluctuation after the warm-up period was provided in the lamp specifications from the vendor to be within 5\%). Finally, after the lamp warm-up period, fiber bending loss was tested by wrapping the detection arm of the probe on a rod 2 and $3 \mathrm{~cm}$ in diameter (D), respectively for three turns each. Five consecutive scans were acquired from the Spectralon within a period of 5 min under each bending diameter (no bending, and $\mathrm{D}=2$ and $3 \mathrm{~cm}$ ). The spectra for $\mathrm{D}=2$ and $3 \mathrm{~cm}$ were compared to the spectra obtained without fiber bending.

\subsection{Wavelength Response of the Calibration Channel}

Although the two channels share the same light source and spectrometer, differences exist in the throughput between the two channels due to wavelength dependence of the splitter and coupling optics, and pixel-to-pixel variations in the CCD efficiency. This can be easily corrected by taking a one-time measurement on a spectrally flat diffuse reflectance standard, such as a Spectralon, and then generating a correction factor $F_{\text {corr }}(\lambda)$ as defined in Eq. (1).

$$
F_{\text {corr }}(\lambda)=R_{\text {Spectralon } 0}(\lambda) / R_{\mathrm{SC} 0}(\lambda),
$$

where $R_{\text {Spectralono }}(\lambda)$ is the diffuse reflectance spectrum measured from the Spectralon and $R_{\mathrm{SC} 0}(\lambda)$ is the self-calibration spectrum collected concurrently.

\subsection{Correction for Lamp Warm-Up}

Of the three systematic errors characterized in Sec. 2.2, warm up introduced the greatest variation in source intensity. The capability of the DRS system for correction of system drift, which is dominated by the lamp intensity variations during warm-up, was evaluated. Specifically, reflectance and self-calibration spectra between 472 and $600 \mathrm{~nm}$ (high scan 
only) were collected from the Spectralon at different time points during instrument warm up. The probe tip was aligned perpendicular to the surface of the Spectralon at a fixed distance $(\sim 1 \mathrm{~mm})$ and maintained at that position throughout the experimental procedure. A calibrated reflectance spectrum was obtained by dividing the raw reflectance spectrum by the self-calibration spectrum collected simultaneously, and then was normalized to the calibrated first scan for variation calculation. The variation in the calibrated reflectance spectrum achieved with the new SC probe B was compared to that achieved with the original SC probe A described in our previous publication. $^{27}$

\subsection{Phantom Experiments}

Tissue-simulating liquid phantoms were utilized to evaluate the performance of the DRS system for measuring tissue optical properties on two different days. The phantoms contained variable concentrations of hemoglobin $(\mathrm{Hb})$ as the absorber and $1-\mu \mathrm{m}$ polystyrene microspheres (07310-15, Polysciences,
Incorporated, Warrington, Pennsylvania.) as the scatterer. Powder form of human $\mathrm{Hb}$ (H0267, Sigma-Aldrich Company, Saint Louis, Missouri) has been widely used as a stable absorber in optical spectroscopy by our group as well as by others. ${ }^{1,28}$ The absorption coefficient $\left(\mu_{a}\right)$ was determined from a spectrophotometer measurement of a diluted $\mathrm{Hb}$ stock solution, and the reduced scattering coefficients $\left(\mu_{s}^{\prime}\right)$ was calculated using the Mie theory ${ }^{29}$ for known size, density, and refractive index of the scatterers.

Two sets of phantoms with identical $\mu_{s}^{\prime}$ were obtained through 17 successive titrations of $\mathrm{Hb}$ from 1 to $31.9 \mu \mathrm{M}$ for day 1 and 0.9 to 29.7 for day 2. Table 1 summarizes the $\mathrm{Hb}$ concentrations and expected phantom optical properties averaged over the wavelength range of 450 to $600 \mathrm{~nm}$. Although the number of scatterers was fixed, the wavelength averaged $\mu_{s}^{\prime}$ decreased from 17.3 to 12.5 with successive dilution. The overall $\mu_{a}$ ranges from 0.016 to $4.65 \mathrm{~cm}^{-1}$ for day 1 and 0.011 to $4.38 \mathrm{~cm}^{-1}$ for day 2 , over 450 to $600 \mathrm{~nm}$.

A diffuse reflectance spectrum was measured with a calibration spectrum concurrently from each phantom using the

Table 1 Summary of the $\mathrm{Hb}$ concentrations and expected phantom optical properties averaged over the wavelength from 450 to $600 \mathrm{~nm}$. Phantom 11 was selected as the reference phantom for all MC inversions.

\begin{tabular}{|c|c|c|c|c|c|}
\hline \multirow[b]{2}{*}{ Phantom } & \multirow[b]{2}{*}{$\begin{array}{l}\text { Averaged } \\
\mu_{s}^{\prime}\left(\mathrm{cm}^{-1}\right)\end{array}$} & \multicolumn{2}{|c|}{ Day 1} & \multicolumn{2}{|c|}{ Day 2} \\
\hline & & $\begin{array}{c}\mathrm{Hb} \\
(\mu \mathrm{M})\end{array}$ & $\begin{array}{l}\text { Averaged } \\
\mu_{a}\left(\mathrm{~cm}^{-1}\right)\end{array}$ & $\begin{array}{c}\mathrm{Hb} \\
(\mu \mathrm{M})\end{array}$ & $\begin{array}{l}\text { Averaged } \\
\mu_{a}\left(\mathrm{~cm}^{-1}\right)\end{array}$ \\
\hline 1 & 17.33 & 1.02 & 0.085 & 0.91 & 0.08 \\
\hline 2 & 17.15 & 2.21 & 0.18 & 2.09 & 0.17 \\
\hline 3 & 16.97 & 3.31 & 0.27 & 3.19 & 0.26 \\
\hline 4 & 16.80 & 4.42 & 0.37 & 4.18 & 0.35 \\
\hline 5 & 16.63 & 5.53 & 0.47 & 5.06 & 0.43 \\
\hline 6 & 16.44 & 6.64 & 0.55 & 6.28 & 0.52 \\
\hline 7 & 16.28 & 7.75 & 0.65 & 7.27 & 0.61 \\
\hline 8 & 16.10 & 8.86 & 0.75 & 8.15 & 0.69 \\
\hline 9 & 15.93 & 9.97 & 0.84 & 9.26 & 0.78 \\
\hline 10 & 15.76 & 11.08 & 0.93 & 10.37 & 0.87 \\
\hline 11 & 15.39 & 13.31 & 1.11 & 12.47 & 1.04 \\
\hline 12 & 15.04 & 15.55 & 1.30 & 14.47 & 1.21 \\
\hline 13 & 14.71 & 17.79 & 1.49 & 16.60 & 1.39 \\
\hline 14 & 14.17 & 21.15 & 1.76 & 19.83 & 1.65 \\
\hline 15 & 13.66 & 24.53 & 2.05 & 22.85 & 1.91 \\
\hline 16 & 13.13 & 27.91 & 2.33 & 25.99 & 2.17 \\
\hline 17 & 12.52 & 31.87 & 2.65 & 29.71 & 2.47 \\
\hline Overall range & 11.56 to 19.15 & 1.02 to 31.87 & 0.016 to 4.65 & 0.91 to 29.71 & 0.011 to 4.38 \\
\hline
\end{tabular}



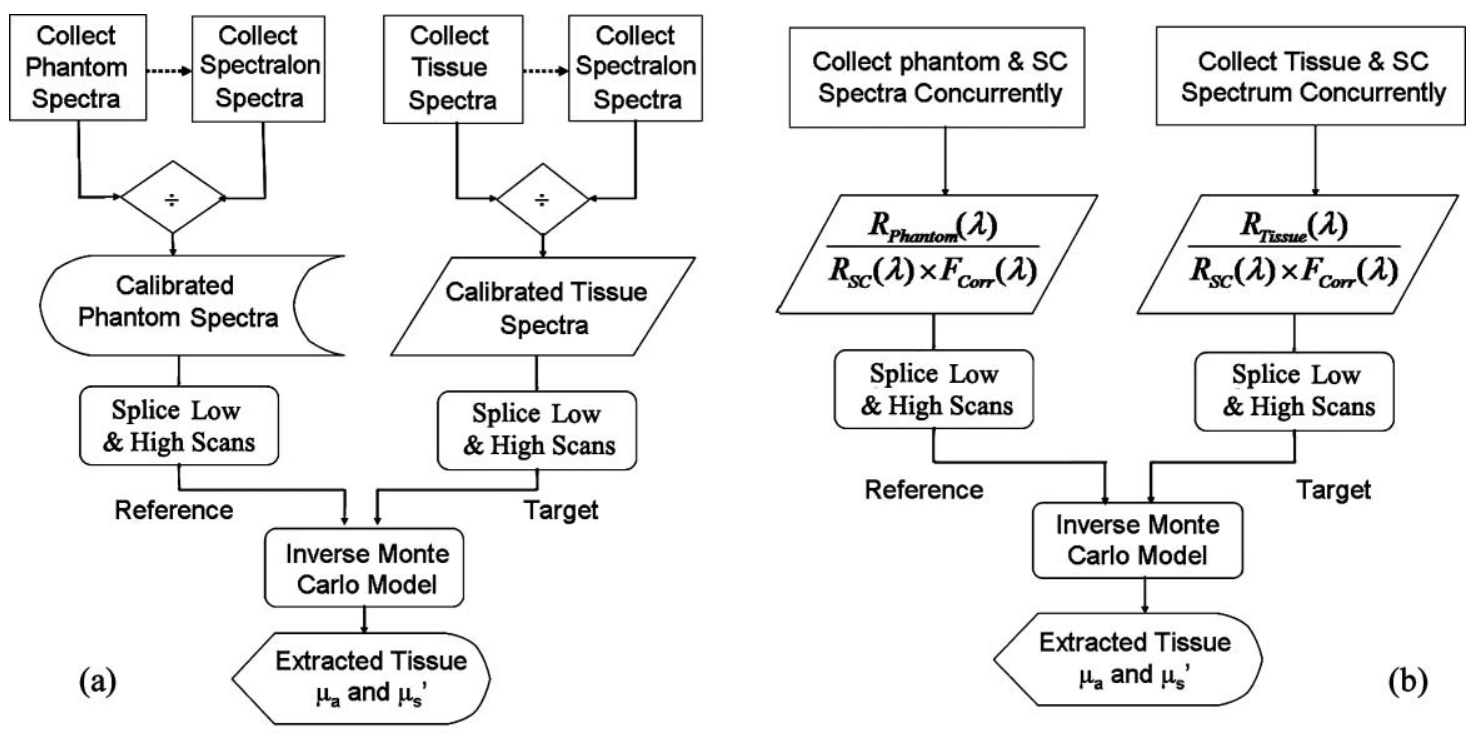

Fig. 2 Data analysis procedures: (a) with Spectralon calibration and (b) with self-calibration.

SC probe B. On day 1 , the measurements were taken $30 \mathrm{~min}$ after the instrument was turned on to eliminate the effect of source intensity variations due to instrument warm-up. On day 2, the measurements started only 2 min after the instrument was turned on to include the effect of source intensity variations due to instrument warm-up. On both days, a reflectance spectrum was also measured from the Spectralon immediately after all the phantom measurements using the same integration times as for the phantom measurements. Approximately 45 to 60 min elapsed between the first phantom and the Spectralon measurements on both days.

\subsection{Data Analysis}

The data analysis procedures for tissue optical spectroscopy are outlined in Fig. 2. In this study, tissue-simulating liquid phantoms were used rather than tissue as the target, and one of the phantoms from the tissue-simulating phantom set was selected as the reference. Hemoglobin, in its oxidized form, was the only absorber in all phantoms.

The first step of the data analysis was calibration, which was performed using two approaches: Spectralon calibration and self-calibration, according to the procedures outlined in Fig. 2. With Spectralon-based calibration, the calibrated spectrum was obtained by dividing the reflectance spectrum (low or high scans) by the corresponding Spectralon measurement (the low or high scans with identical integration time). With selfcalibration, the calibrated spectrum was obtained by dividing the reflectance spectrum by the self-calibration spectrum collected concurrently, and then by the correction factor. In both calibration approaches, a complete calibrated spectrum was assembled by scaling the calibrated low scan to the high scan at $476 \mathrm{~nm}$ (the middle wavelength of overlap between the two scans), and then splicing them into a single spectrum.

Next, the calibrated target tissue phantom spectrum was further normalized to a similarly calibrated reference phantom spectrum prior to analysis by a fast, scalable inverse MC model developed by our group to extract the tissue optical properties. ${ }^{1}$
In this study, phantom 11 was selected as the reference phantom based on a previously published study by our group. ${ }^{28}$ The calibration of the target tissue phantom spectrum against a reference phantom is needed to scale the MC modeled data to the experimentally measured data, while the calibration of the tissue and reference phantom spectra to the Spectralon or SC spectrum is carried out to account for day-to-day or real-time variations in system throughput, respectively.

The inverse MC model of reflectance that was used for extraction of the phantom optical properties consists of a forward model and an inverse model. In the forward model, a set of absorbers are presumed to be present in the medium, and the scatterer is assumed to be single sized, spherically shaped, and uniformly distributed. The wavelength-dependent absorption coefficients of the medium are calculated from the concentration of each absorber and the corresponding wavelength-dependent extinction coefficients. The wavelength-dependent $\mu_{s}^{\prime}$ is calculated from scatterer size, density, and the refractive index of the scatterer and surrounding medium from the Mie theory for spherical particles using freely available software. ${ }^{30}$ The absorption and scattering coefficients are then input into the scalable MC physical model of light transport to obtain a modeled diffuse reflectance spectrum. In the inverse model, the modeled diffuse reflectance is adaptively fitted to the measured tissue diffuse reflectance. When the sum of squares error between the modeled and measured diffuse reflectance is minimized, the concentrations of absorber, scatter size, and density are extracted. An attribute of the scalable MC model of reflectance is that it accounts for the specific probe geometry used for diffuse reflectance measurements. A detailed description of this physical model is provided elsewhere. ${ }^{1}$

Inversions were performed in the wavelength range of 450 to $600 \mathrm{~nm}$ for best signal-to-noise ratio. Two different approached were used: within-day data analysis, in which the target and reference phantoms were from same-day measurements, and across-day data analysis, in which the target and reference phantoms were from different days. The within-day analysis compares the accuracy of the two calibration approaches with and 
without lamp warm-up, while the across-day analysis tests the capability of the self-calibration to correct for day-to-day instrument drift (a larger drift was created by starting phantom measurements during lamp warm-up on day 2), and is representative of what would happen in an actual clinical setting.

\section{Results}

This section first presents the effect of the three types of systematic error on measuring diffuse reflectance spectra, and the implementation of the correction factor for correcting the throughput difference between the tissue and calibration channels. Following these results, the warm up experimental data show that the lamp intensity fluctuations during warm-up can be reduced to a negligible level using the newly designed SC probe $\mathrm{B}$, and finally, the results from the phantom experiments indicate that both real-time and day-to-day instrument drifts can also be effectively corrected by self-calibration.

\subsection{Sources of Systematic Errors}

Figure 3(a) shows ten reflectance spectra from 470 to $600 \mathrm{~nm}$ (high scan only) collected from the same Spectralon on two different days with the experimental setup unchanged. The five consecutive scans on each day (in a single color or solid line) showed very small variations (less than $1 \%$ ). Although the spectra from the two different days have similar line shape, the lamp intensity drift from day 1 to day 2 ranges from 3 to $7 \%$ over the entire wavelength range. This day-to-day lamp intensity fluctuation makes it necessary to have a reproducible calibration measurement before or after every clinical study, which usually takes 5 to $20 \mathrm{~min}$ of valuable time in a clinical setting. Figure 3(b) shows the lamp intensity at two wavelengths, 475 and $575 \mathrm{~nm}$ (normalized to the maximum intensity for $575 \mathrm{~nm}$ ), over a period of $60 \mathrm{~min}$ after the lamp was switched on for two off/on cycles. An increase of 55\% at $575 \mathrm{~nm}$ and $38 \%$ at $475 \mathrm{~nm}$ in the lamp intensity was observed in the first $30 \mathrm{~min}$ for the first cycle. The increases for the second cycle are $38 \%$ at $575 \mathrm{~nm}$ and $24 \% 475 \mathrm{~nm}$. The intensity fluctuation was less than $5 \%$ between 30 and $60 \mathrm{~min}$, which agrees with the lamp specification provided by the vendor. The source intensity variations are large, as shown in Fig. 3(b), due to the rapid increase in the lamp intensity at the early time points. The difference in the intensity change at the two wavelengths in the first $30 \mathrm{~min}$ also indicates that the line shape of the lamp spectrum changes over time during warm up and thus cannot be accounted for with a simple one-time calibration measurement. This source of error is currently accounted for by allowing the instrument to warm up for a certain period of time (30 $\mathrm{min})$ prior to initiating the spectroscopic measurements. However, this assumes that the warm-up period stays the same each time, which may not always be a reasonable assumption. Figure 3(c) shows three sets of spectra (five in each color or solid line) that were collected between 470 and $600 \mathrm{~nm}$ (high scan only) for the case where there was no bending, a bending diameter of $\mathrm{D}=3$, and a bending diameter of $2 \mathrm{~cm}$, respectively. Obviously, the bending-induced attenuation is significantly higher than the repeated measurements or day-to-day variations. An average fiber bending loss of 8.7 and $68.9 \%$ was observed at $470 \mathrm{~nm}$ for $\mathrm{D}=3$ and $2 \mathrm{~cm}$, respectively. The sharp increase in the bending loss at $\mathrm{D}=2 \mathrm{~cm}$ indicates that there was

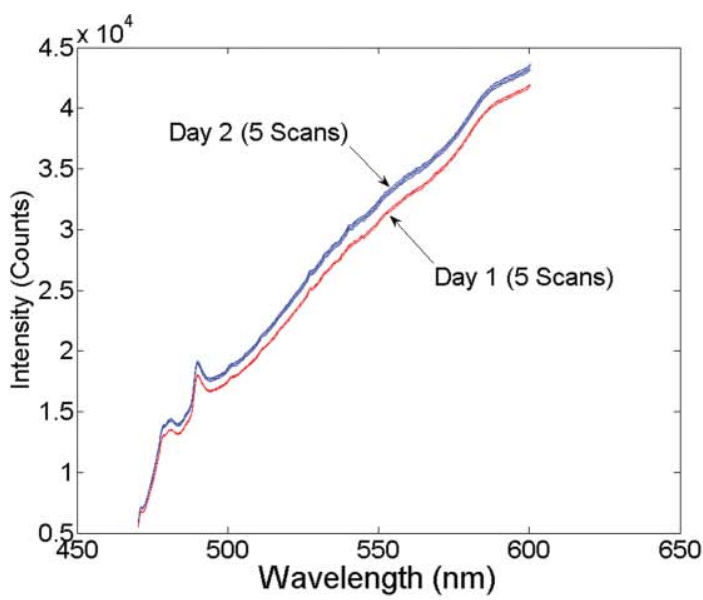

(a)

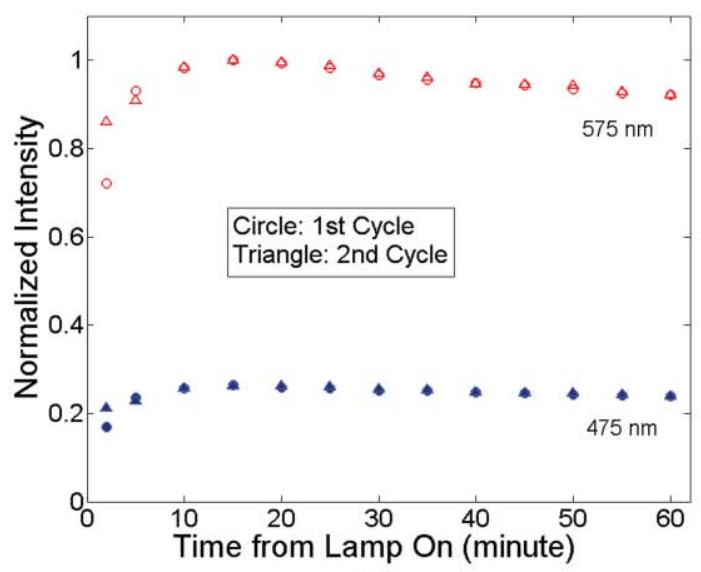

(b)



(c)

Fig. 3 Sources of instrumental errors. (a) Day-to-day lamp intensity fluctuations measured with SC probe B. Each color represents five consecutive scans per day from each of two days. (b) Lamp intensity fluctuations during a 60-min warm-up period normalized to the maximum intensity at $575 \mathrm{~nm}$. (c) Intensity attenuation induced by fiber bending loss measured with SC probe B. Each color represents five consecutive scans under the same bending diameter. All of these measurements were made on a Spectralon reflectance standard with high scan only. (Color online only.) 
a significant increase in wave coupling from the guided modes to cladding (leaky) modes in the fibers. There is, to the best of our knowledge, no existing technology to correct for bending loss that occurs during a tissue or phantom measurement.

\subsection{Correction Factor}

Figure 4(a) shows the raw reflectance spectrum from a Spectralon and the self-calibration spectrum collected concurrently after 30 min of lamp warm-up. The SC channel has a different wavelength response compared with the tissue channel, due to the use of a stainless-steel reflective rod and the free-space coupling from the calibration source fiber to the return fiber, as illustrated in Fig. 1(b). Thus a correction factor for this wavelengthdependent response was generated [defined in Eq. (1)] for this particular probe and is shown in Fig. 4(b). The relatively large fluctuations between 450 and $500 \mathrm{~nm}$ of the correction factor could be due to multiple emission peaks in the lamp spectrum and the optical aberrations in the spectrometer, which might result in different wavelength scales for the two channels. It could also be caused by the splicing of the two scans that is required to cover a wavelength range from 350 to $600 \mathrm{~nm}$ using this instrument. However, the fluctuations should not affect the accuracy of the calibration as long as it is repeatable. The repeatability of the correction factor was measured by reattaching (10 times) the probe to the instrument, which may be required for probe sterilization, and the coefficient of variation (COV) was plotted in Fig. 4(c). The COV averaged over the wavelength range of 350 to $600 \mathrm{~nm}$ is $1.35 \%$. The largest variation appears at the wavelength below $450 \mathrm{~nm}$ due to relatively low lamp intensity. In this work, spectral analysis of the phantom data was performed between 450 and $600 \mathrm{~nm}$. The variation is mainly due to the probe adaptor design, which uses a setscrew to lock the collection fiber bundle into a tube on the instrument side and can be further reduced by alternate designs. The correction factor was saved in the computer and was used for subsequent calibrations using the procedures shown in Fig. 2(b). Each instrument and probe combination has its own wavelength-dependent response and requires a unique correction factor $F_{\text {corr }}(\lambda)$. Using SC probe $B$ with the correction factor, the sources of errors presented in Figs. 3(a), 3(b), and 3(c) can be corrected in real-time.

\subsection{Correction for Lamp Intensity Fluctuations}

Figure 5(a) shows the raw reflectance spectra (high scans only) that were collected by SC probe B from the Spectralon at successive time points starting 2 min from when the lamp was turned on. The lamp intensity increased by 14 to $20 \%$ from 2 to $40 \mathrm{~min}$, which is slightly lower than that measured in Fig. 3(b). What needs to be mentioned is that the spectra in Figs. 3(a), 3(c), and 5(a) were collected in three independent experiments, in which probe-to-Spectralon coupling and fiber bending were not repeatable. Therefore, the spectral intensities varied significantly among the three experiments. The dips around $542.5 \mathrm{~nm}$ in Fig. 5(a) could be due to a bad pixel on the CCD chip or a tiny crack in one of the collection fibers. Figure 5(b) shows the raw reflectance spectra divided by the calibration spectra collected concurrently and then normalized to the first scan. Dividing the raw spectra by the calibration spectra reduced the intensity fluctuations down to $\pm 1 \%$ (except for the edge pixels, which were

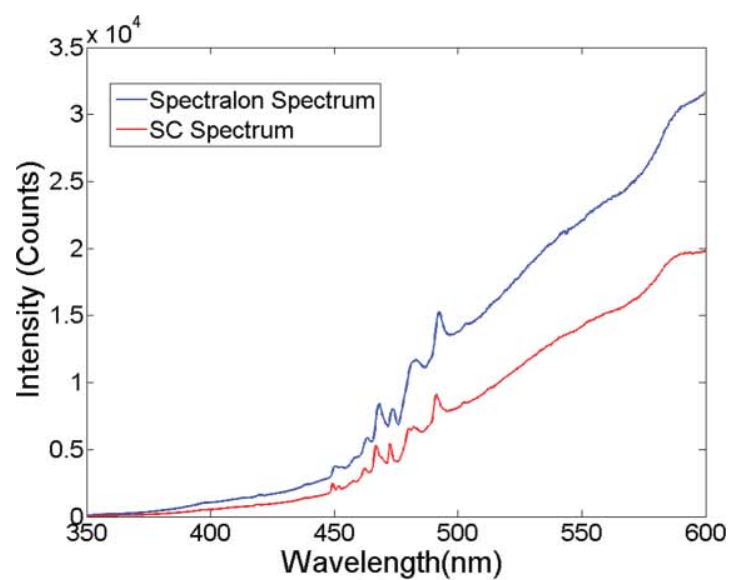

(a)



(b)



(c)

Fig. 4 Correction for wavelength dependence of the self-calibration channel between 350 and $600 \mathrm{~nm}$ (obtained from two scans): (a) raw spectra; (b) correction factor $F_{\text {corr }}(\lambda)$; and (c) coefficient of variation of $F_{\text {corr }}(\lambda)$ in 10 repeated experiments.

discarded during the splicing), demonstrating that the lamp fluctuations shown in Fig. 3(b) can be corrected by self-calibration. This also indicates that the repeatability of the correction factor is within $\pm 1 \%$. As a comparison, Figs. 5(c) and 5(d) show the raw and normalized self-calibrated reflectance spectra obtained using the SC probe A (with separate illumination fibers for 



Fig. 5 Correction for lamp intensity variations: (a) raw spectra from the Spectralon at various time points after the lamp was turned on, and (b) ratio between the Spectralon and self-calibration spectra normalized to the first scan using SC probe B. (c) Raw spectra from the Spectralon at various time points after the lamp was turned on, and (d) ratio between the Spectralon and self-calibration spectra normalized to the first scan using SC probe A.

tissue and calibration channels) at successive time points starting $5 \mathrm{~min}$ from when the lamp was turned on. Even with smaller lamp drifts $(\sim 8 \%$, resulting from the slightly longer warm-up time for the first measurement), the calibrated spectra still show a $4 \%$ variation, which is likely due to variation of the spatial distribution of the lamp emission during warm up.

\subsection{Phantom Experimental Results}

The Spectralon- and self-calibrated reflectance spectra measured from phantoms 1 (lightest), 9 (medium), and 17 (darkest), all from day 1 , are plotted over the full wavelength range from 350 to $600 \mathrm{~nm}$ in Fig. 6. The three intensity valleys at 415, 540, and $575 \mathrm{~nm}$ in all spectra correspond to the Soret band (400 to $450 \mathrm{~nm}), \alpha$ band $(540 \mathrm{~nm})$, and $\beta$ band (569 nm) of oxygenated hemoglobin, respectively. The absorption of the $\alpha$ and $\beta$ bands in phantom 1 are hardly noticeable due to the low $\mathrm{Hb}$ concentration in this phantom. The self-calibrated and Spectralon calibrated spectra for phantoms 9 and 17 show excellent overlap. The small but distinct difference between the two calibrated spectra for phantom 1 is likely caused by instrument drift during the time elapsed ( $>45 \mathrm{~min}$ ) between the first phantom measurement and Spectralon measurement that was performed right after phantom 17.

\subsubsection{Within-day data analysis}

In the within-day data analysis, the MC inversions were performed between the wavelengths of 450 and $600 \mathrm{~nm}$ for all target phantom spectra and reference phantom (phantom 11) from the same day. The extracted versus expected $\mathrm{Hb}$ concentrations



Fig. 6 Spectralon- and self-calibrated phantom spectra for phantoms 1,9 , and 17 , all from day 1. 




(a)

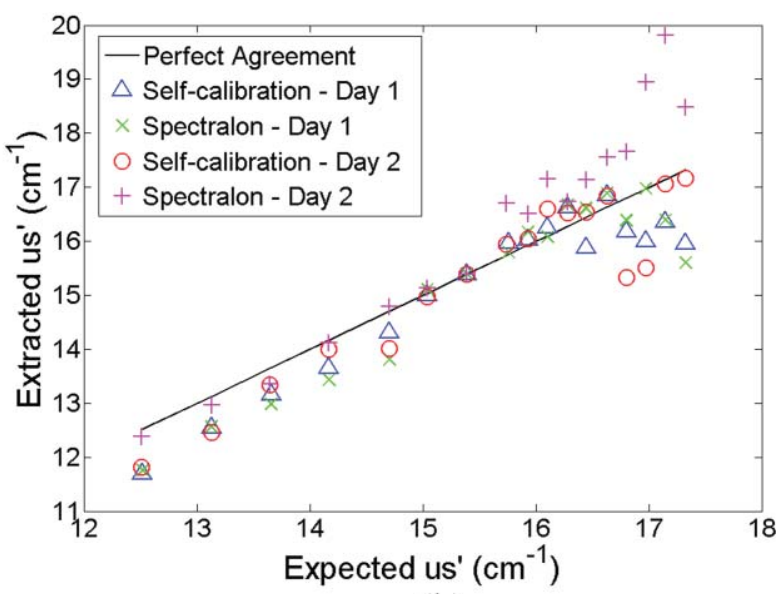

(b)

Fig. 7 Extracted versus expected: (a) Hb concentrations and (b) wavelength-averaged reduced scattering coefficients over 450 to $600 \mathrm{~nm}$ from within-day data analysis, in which phantom 11 from the same day was used as a reference.

and wavelength-averaged reduced scattering coefficients over 450 to $600 \mathrm{~nm}$ are plotted in Figs. 7(a) and 7(b), respectively. On either day, both the self- and Spectralon-calibration methods resulted in excellent agreement for the extracted and expected $\mathrm{Hb}$ concentrations (overall error within $8.8 \%$ ) as well as for the reduced scattering coefficients (overall error within $4.5 \%$ ). On day 2 , in which phantom measurements were made during the lamp warm-up period, however, the extracted reduced scattering coefficients with the Spectralon calibration diverged considerably from the expected values at higher $\mu_{s}^{\prime}$, which corresponds to the first few phantoms, and the overall error is higher than the other three sets shown in Fig. 7(b). This is due to the fact that Spectralon calibration, which was performed after the phantom studies, did not account for the variations in source intensity due to lamp warm up.

\subsubsection{Across-day data analysis}

In the across-day data analysis, MC inversions were first performed using all phantoms from day 1 as targets and phantom
11 from day 2 as the reference (referred to as day 1/2), and then all phantoms from day 2 as targets and phantom 11 from day 1 as the reference (referred to as day 2/1). As a comparison, both Spectralon- and self-calibrated phantom spectra were used for the inversions. The extracted versus expected $\mathrm{Hb}$ concentrations and wavelength-averaged reduced scattering coefficients are presented in Fig. 8. With either calibration approach and target-reference combination (day $1 / 2$ or $2 / 1$ ), the extracted $\mathrm{Hb}$ concentrations show excellent agreement with the expected values. However, in either target-reference combination, the extracted reduced-scattering coefficients with self-calibration have significantly better agreement with expected values than those with the traditional Spectralon-based calibration.

Table 2 summarizes the errors averaged across all the target phantoms obtained with the two calibration techniques and data analysis strategies: within-day data analysis (columns day $1 / 1$ and 2/2) and across-day data analysis (day $1 / 2$ and 2/1). Again, the accuracy for $\mathrm{Hb}$ concentration extraction for day $1 / 1,2 / 2$, and $1 / 2$ is comparable between the two calibration techniques using either data analysis method, indicating that it

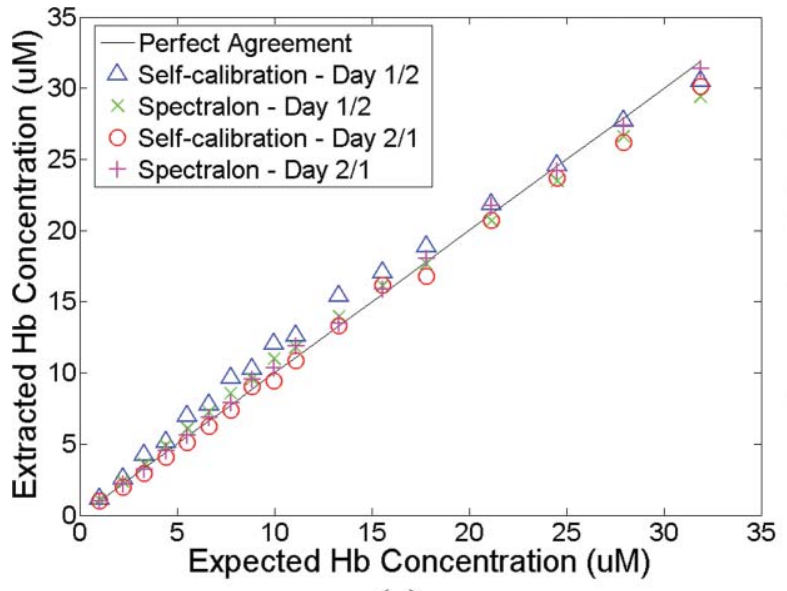

(a)

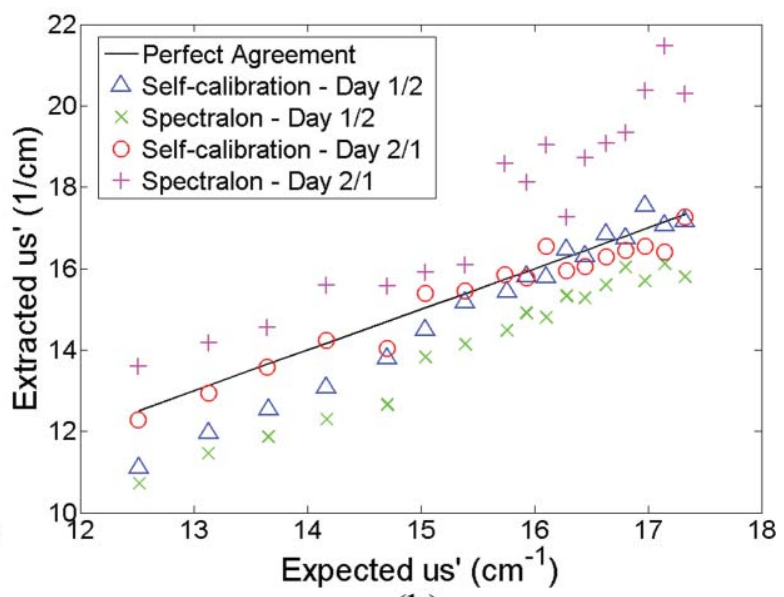

(b)

Fig. 8 Extracted versus expected: (a) Hb concentrations and (b) wavelength-averaged reduced scattering coefficients from across-day data analysis, in which phantom 11 from a different day was used as a reference. 
Table 2 Errors in extraction of phantom optical properties in the wavelengths range of 450 to $600 \mathrm{~nm}$.

\begin{tabular}{|c|c|c|c|c|c|c|c|c|}
\hline \multirow[b]{2}{*}{ Target/reference } & \multicolumn{4}{|c|}{ Spectralon calibration } & \multicolumn{4}{|c|}{ Self-calibration } \\
\hline & Day $1 / 1$ & Day $2 / 2$ & Day $1 / 2$ & Day $2 / 1$ & Day $1 / 1$ & Day $2 / 2$ & Day 1 /2 & Day $2 / 1$ \\
\hline Error in $\mathrm{Hb}$ concentration & $6.9 \pm 8.1 \%$ & $8.8 \pm 4.5 \%$ & $8.4 \pm 4.9 \%$ & $4.5 \pm 1.5 \%$ & $8.5 \pm 8.6 \%$ & $7.6 \pm 8.1 \%$ & $7.0 \pm 4.4 \%$ & $8.6 \pm 3.9 \%$ \\
\hline Error in $\mu_{s}^{\prime}$ & $3.0 \pm 2.8 \%$ & $4.5 \pm 4.2 \%$ & $9.0 \pm 3.2 \%$ & $12.5 \pm 6.1 \%$ & $3.2 \pm 2.3 \%$ & $2.8 \pm 2.8 \%$ & $3.8 \pm 3.4 \%$ & $2.1 \pm 1.1 \%$ \\
\hline
\end{tabular}

was not significantly affected by the lamp warm-up or day-today instrument drifts. The error for $\mathrm{Hb}$ extraction for day $2 / 1$ with self-calibration is slightly higher than that obtained with Spectralon-calibration, but is still comparable with those obtained with within-day data analysis. However, with Spectralon calibration, the errors for extraction of $\mu_{s}^{\prime}$ are higher on day 2 using within-day data analysis and are remarkably higher using across-day data analysis, compared to the errors obtained with self-calibration. This demonstrates that scattering is more susceptible to lamp intensity fluctuations during warm-up or day-to-day instrument drifts.

\section{Discussion}

The long-term goal of this work is to develop a fiber optic probebased spectrometer for performing instrument-independent diffuse reflectance spectroscopy of human tissue in vivo. We have demonstrated the feasibility of performing DRS in the visible wavelength range using a compact self-calibration fiber optic probe. We showed that with an improved self-calibration probe (SC probe $\mathrm{B}$ ) relative to the one previously published by our group (SC probe A), ${ }^{27}$ the lamp intensity fluctuation during warm-up was successfully reduced from $20 \%$ down to $1 \%$ [Figs. 5(a) and 5(b)]. As a comparison, SC probe A could only reduce the lamp warm-up variations from $8 \%$ (smaller change due to longer warm-up time for the first measurement) to $4 \%$. We also showed, with single- and multiday phantom studies during and after instrument warm-up, that self-calibration results showed comparable accuracy in extraction of absorber concentrations, but significantly improved accuracy in quantifying reduced scattering coefficients, compared with traditional calibration using a reflectance standard, thus more accurately accounting for source intensity fluctuations associated with both instrument warm-up and different-day measurements. The ability to self-calibrate effectively during lamp warm-up is an important advance, since this could significantly reduce the time needed for calibration prior to a clinical measurement. Also, given the unpredictable nature of source intensity fluctuations during a clinical procedure (bumping the instrument or probe, bending, etc.), having a calibration measurement that can account for these real-time source intensity fluctuations through this improved probe design is imperative.

To evaluate the performance of the self-calibration fiber optic probe, we conducted phantom experiments to simulate various scenarios from a practical clinical setting. The first phantom experiment was performed after the instrument was fully warmed up for more than $30 \mathrm{~min}$ and a calibration measurement from a Spectralon standard was taken after all phantom measurements were completed. This simulates the way a clinical DRS study is typically performed with traditional calibration. The second phantom experiment was performed on a different day and during the lamp warm-up period, which was used to test the feasibility of starting a clinical study right after the instrument is turned on. While the within-day data analysis tested the accuracy of the technology without having to consider day-to-day instrument differences or instrument warm up, the across-day analysis tested the accuracy in a more practical situation, which includes both instrument warm-up and day-to-day differences.

We found that the accuracy for extracting $\mathrm{Hb}$ concentration was very consistent (mean errors within 4.5 to $8.8 \%$ ) under different experimental conditions (with and without warm up), with different calibration techniques (SC versus Spectralon), and using different data analysis schemes (within- versus across-day) [Figs. 7(a) and 8(a) and row 3 in Table 2]. This indicates that the extraction of absorption coefficients (or absorber concentrations) was not significantly affected by the lamp warm-up and day-to-day source intensity fluctuations. On the other hand, with Spectralon-based calibration, the errors for extraction of the reduced scattering coefficient are 50\% higher in day 2 than those in day 1 using within-day analysis (day $2 / 2$ versus $1 / 1$ in Table 2), whereas the errors obtained using across-day analysis are about three times higher than those obtained using within day analysis (day $1 / 2$ versus $1 / 1$ or day $2 / 1$ versus $2 / 2$ in Table 2). We believe that the lamp intensity fluctuations had a greater effect on scattering than absorption due to the fact that the lamp intensity mostly affected the overall intensity, but maintained the same emission spectral shape, as shown in Figs. 3(a) and 5(a). One would also expect that the errors would be higher on day $2 / 2$ due to the lamp warm-up effects. Although the Spectralon measurement was taken at the end of the study when the lamp became stable and not able to correct for the lamp intensity changes that occurred during the phantom measurements, the calibration to reference phantom 11 partially reduced the effect of the source fluctuation, as phantom 11 was measured in the middle of the phantom study (between phantom 1 measurement and the Spectralon measurement, presumably after the instrument had warmed up). As indicated in Table 2, the use of self-calibration significantly reduced the errors for extraction of reduced scattering coefficients, which demonstrated the feasibility of performing diffuse reflectance spectroscopy during lamp warm-up and with a reference phantom spectrum measured on different days. It is well known that absorption and scattering coefficients of tissues reflect their underlying physiological and morphological properties, respectively. For example, in the UV and visible band, dominant absorbers in epithelial tissues, where most cancers originate, are oxygenated and deoxygenated hemoglobin, arising from blood vessels in the stromal layer, while light scattering is primarily caused by cell nuclei 
and organelles in the epithelium and stroma, as well as collagen fibers and cross-links in stroma. Neoplastic tissues exhibit significant changes both in their physiological and morphological characteristics: stromal layer absorption is expected to increase with angiogenesis, whereas stromal scattering is expected to go down with neoplastic progression as extracellular collagen networks degrade. ${ }^{2,16,31-34}$ Epithelial scattering has been shown to increase due to increased nuclear size, increased DNA content, and hyperchromasia. ${ }^{2,31,33,35}$ Therefore, being able to accurately quantify tissue scattering properties is as important as being able to quantify as absorption for cancer diagnostics and therapeutics.

Another benefit of the SC probe is its potential for correcting fiber bending loss that can occur during a tissue spectroscopic measurement. We have experimentally investigated this capability of the SC probe. We found that both the previously reported SC probe A and SC probe B described in this work could significantly reduce bending losses at bending diameters more than $3 \mathrm{~cm}$, but were very inefficient when the bending diameter was less than $3 \mathrm{~cm}$. For SC probe A, this is likely due to the different mode excitation at the distal end between the tissue channel (diffuse reflectance) and the calibration channel (simple specular reflection), thus resulting in different responses to fiber bending. For SC probe B, in addition to the different mode excitations at the distal end of the two channels, the coupling ratio of the multimode splitter is also sensitive to the mode distributions inside the fibers and may change with fiber bending. At a small bending diameter, the coupling among different fiber modes becomes comparable to or even higher than the bending loss itself. Although a specification on the minimum bending diameter for the fibers was not provided by the manufacturers, a minimum bending radius of $2 \mathrm{~cm}$ for $200 \mu \mathrm{m}$ and $3 \mathrm{~cm}$ for $400 \mu \mathrm{m}$ high-OH fibers is recommended by Ocean Optics, Incorporated (Dunedin, Florida). The bending radii we have tested are slightly smaller than the suggested minimum bending radii. However, in practice, particularly in clinical studies, the focus of the operators will be on the patient, and the fiber cables are often accidently bent much smaller than this minimum radius. We believe it is important to correct for bending, particularly for larger bending radii. To improve the efficiency for bending correction, multiple strategies may be required in building the next-generation probe, and these strategies will be investigated in our future work.

Although we have only investigated the use of the selfcalibration probe for UV-VIS diffuse reflectance spectroscopy, the self-calibration technique can be readily extended to other wavelength ranges, such as in the near-infrared. The technique can potentially be adopted for fluorescence or Raman spectroscopy, in which the calibration channel will provide realtime calibration information for the lamp power and excitation throughput.

\section{Conclusions}

We demonstrate the feasibility of performing UV-VIS diffuse reflectance spectroscopy with a self-calibration fiber optic probe. Combined with a one-time, single-reference phantom measurement, the self-calibration probe can provide instrumentindependent optical properties of in-vivo tissue using optical spectroscopy. Compared with conventional calibration tech- niques, self-calibration makes the calibration process easier, real time, more robust, and faster, and could potentially save up to $60 \mathrm{~min}$ of precious clinical time for in-vivo studies.

\section{Acknowledgments}

This work has been funded by National Institutes of Health (NIH) grant R01CA100559-01A1, and DOD BCRP Era of Hope award to Ramanujam.

\section{References}

1. G. M. Palmer and N. Ramanujam, "Monte Carlo-based inverse model for calculating tissue optical properties. Part I: theory and validation on synthetic phantoms," Appl. Opt. 45(5), 1062-1071 (2006).

2. D. Arifler, R. A. Schwarz, S. K. Chang, and R. Richards-Kortum, "Reflectance spectroscopy for diagnosis of epithelial precancer: modelbased analysis of fiber-optic probe designs to resolve spectral information from epithelium and stroma," Appl. Opt. 44(20), 4291-4305 (2005).

3. P. Thueler, I. Charvet, F. Bevilacqua, M. St Ghislain, G. Ory, P. Marquet, P. Meda, B. Vermeulen, and C. Depeursinge, "In vivo endoscopic tissue diagnostics based on spectroscopic absorption, scattering, and phase function properties," J. Biomed. Opt. 8(3), 495-503 (2003).

4. Q. Wang, H. Yang, A. Agrawal, N. S. Wang, and T. J. Pfefer, "Measurement of internal tissue optical properties at ultraviolet and visible wavelengths: Development and implementation of a fiber optic-based system," Opt. Express 16(12), 8685-8703 (2008).

5. G. Zonios, L. T. Perelman, V. Backman, R. Manoharan, M. Fitzmaurice, J. Van Dam, and M. S. Feld, "Diffuse reflectance spectroscopy of human adenomatous colon polyps in vivo," Appl. Opt. 38(31), 6628-6637 (1999).

6. T. J. Farrell, M. S. Patterson, and B. Wilson, "A diffusion theory model of spatially resolved, steady-state diffuse reflectance for the noninvasive determination of tissue optical properties in vivo," Med. Phys. 19(4), 879-888 (1992).

7. Z. Volynskaya, A. S. Haka, K. L. Bechtel, M. Fitzmaurice, R. Shenk, N. Wang, J. Nazemi, R. R. Dasari, and M. S. Feld, "Diagnosing breast cancer using diffuse reflectance spectroscopy and intrinsic fluorescence spectroscopy," J. Biomed. Opt. 13(2), 024012 (2008).

8. U. Utzinger, M. Brewer, E. Silva, D. Gershenson, R. C. Blast, Jr., M. Follen, and R. Richards-Kortum, "Reflectance spectroscopy for in vivo characterization of ovarian tissue," Lasers Surg. Med. 28(1), 56-66 (2001).

9. Y. N. Mirabal, S. K. Chang, E. N. Atkinson, A. Malpica, M. Follen, and R. Richards-Kortum, "Reflectance spectroscopy for in vivo detection of cervical precancer," J. Biomed. Opt. 7(4), 587-594 (2002).

10. K. Badizadegan, V. Backman, C. W. Boone, C. P. Crum, R. R. Dasari, I. Georgakoudi, K. Keefe, K. Munger, S. M. Shapshay, E. E. Sheetse, and M. S. Feld, "Spectroscopic diagnosis and imaging of invisible pre-cancer," Faraday Discuss. 126, 265-279; discussion 303-211 (2004).

11. R. A. Schwarz, W. Gao, D. Daye, M. D. Williams, R. Richards-Kortum, and A. M. Gillenwater, "Autofluorescence and diffuse reflectance spectroscopy of oral epithelial tissue using a depth-sensitive fiber-optic probe," Appl. Opt. 47(6), 825-834 (2008).

12. N. M. Marin, A. Milbourne, H. Rhodes, T. Ehlen, D. Miller, L. Benedet, R. Richards-Kortum, and M. Follen, "Diffuse reflectance patterns in cervical spectroscopy," Gynecol. Oncol. 99(3 Suppl 1), S116-120 (2005).

13. C. Zhu, G. M. Palmer, T. M. Breslin, J. Harter, and N. Ramanujam, "Diagnosis of breast cancer using diffuse reflectance spectroscopy: comparison of a Monte Carlo versus partial least squares analysis based feature extraction technique," Lasers Surg. Med. 38(7), 714-724 (2006).

14. M. C. Skala, G. M. Palmer, K. M. Vrotsos, A. Gendron-Fitzpatrick, and N. Ramanujam, "Comparison of a physical model and principal component analysis for the diagnosis of epithelial neoplasias in vivo using diffuse reflectance spectroscopy," Opt. Express 15(12), 78637875 (2007) 
15. A. Sharwani, W. Jerjes, V. Salih, B. Swinson, I. J. Bigio, M. El-Maaytah, and C. Hopper, "Assessment of oral premalignancy using elastic scattering spectroscopy," Oral Oncol. 42(4), 343-349 (2006).

16. M. G. Muller, T. A. Valdez, I. Georgakoudi, V. Backman, C. Fuentes, S. Kabani, N. Laver, Z. Wang, C. W. Boone, R. R. Dasari, S. M. Shapshay, and M. S. Feld, "Spectroscopic detection and evaluation of morphologic and biochemical changes in early human oral carcinoma," Cancer 97(7), 1681-1692 (2003).

17. A. Amelink, O. P. Kaspers, H. J. Sterenborg, J. E. Van Der Wal, J. L. Roodenburg, and M. J. Witjes, "Non-invasive measurement of the morphology and physiology of oral mucosa by use of optical spectroscopy," Oral Oncol. 44(1), 65-71 (2008).

18. L. T. Nieman, C. W. Kan, A. Gillenwater, M. K. Markey, and K. Sokolov, "Probing local tissue changes in the oral cavity for early detection of cancer using oblique polarized reflectance spectroscopy: a pilot clinical trial," J. Biomed. Opt. 13(2), 024011 (2008).

19. I. J. Bigio and S. G. Bown, "Spectroscopic sensing of cancer and cancer therapy: current status of translational research," Cancer Biol. Ther. 3(3), 259-267 (2004).

20. W. C. Lin, S. A. Toms, M. Motamedi, E. D. Jansen, and A. MahadevanJansen, "Brain tumor demarcation using optical spectroscopy; an in vitro study," J. Biomed. Opt. 5(2), 214-220 (2000).

21. J. Q. Brown, T. M. Bydlon, L. M. Richards, B. Yu, S. A. Kennedy, J. Geradts, L. G. Wilke, M. Junker, J. Gallagher, and N. Ramanujam, "Optical assessment of tumor resection margins in the breast," IEEE J. Sel. Topics Quantum Electron. 16, 530-544 (2010).

22. U. Sunar, H. Quon, T. Durduran, J. Zhang, J. Du, C. Zhou, G. Yu, R. Choe, A. Kilger, R. Lustig, L. Loevner, S. Nioka, B. Chance, and A. G. Yodh, "Noninvasive diffuse optical measurement of blood flow and blood oxygenation for monitoring radiation therapy in patients with head and neck tumors: a pilot study," J. Biomed. Opt. 11(6), 064021 (2006).

23. H. W. Wang, T. C. Zhu, M. E. Putt, M. Solonenko, J. Metz, A. Dimofte, J. Miles, D. L. Fraker, E. Glatstein, S. M. Hahn, and A. G. Yodh, "Broadband reflectance measurements of light penetration, blood oxygenation, hemoglobin concentration, and drug concentration in human intraperitoneal tissues before and after photodynamic therapy," J. Biomed. Opt. 10(1), 14004 (2005).

24. A. Amelink, A. Van Der Ploeg Van DenHeuvel, W. J. de Wolf, D. J. Robinson, and H. J. Sterenborg, "Monitoring PDT by means of superficial reflectance spectroscopy," J. Photochem. Photobiol. B 79(3), 243-251 (2005)

25. P. G. Maxim, J. J. Carson, D. A. Benaron, B. W. Loo, Jr., L. Xing, A. L. Boyer, and S. Friedland, "Optical detection of tumors in vivo by visible light tissue oximetry," Technol. Cancer Res. Treat. 4(3), 227234 (2005).

26. G. M. Palmer, C. Zhu, T. M. Breslin, F. Xu, K. W. Gilchrist, and N. Ramanujam, "Monte Carlo-based inverse model for calculating tissue optical properties. Part II: application to breast cancer diagnosis," Appl. Opt. 45(5), 1072-1078 (2006).

27. B. Yu, H. Fu, T. Bydlon, J. E. Bender, and N. Ramanujam, "Diffuse reflectance spectroscopy with a self-calibrating fiber optic probe," Opt. Lett. 33(16), 1783-1785 (2008).

28. J. E. Bender, K. Vishwanath, L. K. Moore, J. Q. Brown, V. Chang, G. M. Palmer, and N. Ramanujam, "A robust Monte Carlo model for the extraction of biological absorption and scattering in vivo," IEEE Trans. Biomed. Eng. 56(4), 960-968 (2009).

29. S. Prahl, "Mie scattering program," Oregon Med. Laser Center, see http://omlc.ogi.edu/software/mie/index.html (2005).

30. S. Prahl., "Mie scattering program," Oregon Med. Laser Center, Portland, OR (2005).

31. P. M. Lane, T. Gilhuly, P. Whitehead, H. Zeng, C. F. Poh, S. Ng, P. M. Williams, L. Zhang, M. P. Rosin, and C. E. MacAulay, "Simple device for the direct visualization of oral-cavity tissue fluorescence," J. Biomed. Opt. 11(2), 024006 (2006).

32. I. Pavlova, C. R. Weber, R. A. Schwarz, M. D. Williams, A. M. Gillenwater, and R. Richards-Kortum, "Fluorescence spectroscopy of oral tissue: Monte Carlo modeling with site-specific tissue properties," J. Biomed. Opt. 14(1), 014009 (2009).

33. A. Wang, V. Nammalavar, and R. Drezek, "Targeting spectral signatures of progressively dysplastic stratified epithelia using angularly variable fiber geometry in reflectance Monte Carlo simulations," J. Biomed. Opt. 12(4), 044012 (2007).

34. R. Hasina and M. W. Lingen, "Angiogenesis in oral cancer,"J. Dent. Educ. 65(11), 1282-1290 (2001).

35. I. Georgakoudi, E. E. Sheets, M. G. Muller, V. Backman, C. P. Crum, K. Badizadegan, R. R. Dasari, and M. S. Feld, "Trimodal spectroscopy for the detection and characterization of cervical precancers in vivo," Am. J. Obstet. Gynecol. 186(3), 374-382 (2002). 